partners did so. In multivariate analysis, compared with those with 2 partners, those with 3 partners and those with 4 were not significantly more likely to perceive themselves at HSR. At 5 partners the Odds Ratio (OR) was 5.30 (95\% CI 2.30-12.22) and at $\geq 6$ partners it was 9.18; (95\% CI 4.42-19.09). FLASRT was also associated with perceived higher risk (OR 1.07; 95\% CI 1.02-1.13). CK was not associated with risk perception.

Conclusion Higher risk university students often do not recognise their STD risk status, and more often perceive risk only after a high threshold of multiple partners is reached. Prevention messages should emphasise that STD risk exists at lower levels of multiple partners than students perceive.

\section{P4.082 UNDERSTANDING THE LINK BETWEEN ALCOHOL USE AND STIS: TWO TESTS OF MEDIATION}

doi:10.1136/sextrans-2013-051184.0980

'K B Carey, ${ }^{2} \mathrm{~T}$ E Senn, ${ }^{3} \mathrm{P}$ Coury-Doniger, ${ }^{3} \mathrm{M}$ A Urban, ${ }^{2} \mathrm{M}$ P Carey. ${ }^{1}$ Brown University, Providence, Rl, United States; ${ }^{T}$ The Miriam Hospital, Providence, RI, United States; ${ }^{3}$ University of Rochester, Rochester, NY, United States

Background Alcohol use has been associated with STIs. This association is generally attributed to an impaired ability to appreciate future consequences, leading individuals to engage in unprotected sex after consuming alcohol. However, a second pathway through which alcohol use leads to STI is through an impaired ability to use a condom correctly. Problems related to using condoms reduce their efficacy and have been associated with incident STIs. This study investigates both unprotected sex and incorrect condom use as potential mediating pathways through which alcohol use might lead to STIs.

Methods Participants (498 patients; 41\% female; 69\% African American) attending a publicly-funded STI clinic completed a computerised survey assessing global alcohol use (AUDIT-C), alcohol use before sex, incorrect condom use, episodes of unprotected sex, and recent STI history. Bootstrapped mediation analysis with 5000 resamples tested a multiple mediator model in which the number of episodes of unprotected sex and incorrect condom use mediated the relation between alcohol use and STI.

Results Participants reported an average of 17 episodes of unprotected sex in the past 3 months; $17 \%$ reported being diagnosed with an STI in the past 3 months. Controlling for gender, inconsistent condom use mediated the relation between alcohol use before sex and STI (95\% bootstrapped CI for the indirect effect $=0.002$ to $0.056 ; p<0.05)$. The number of episodes of unprotected sex did not mediate this relation. No significant associations were found when global alcohol use was used as the predictor.

Conclusion Alcohol use before sex may increase risk for STIs due to an impaired ability to use condoms correctly, rather than through unprotected sex. Findings have implications for STI prevention because intoxication may impair effective use of condoms despite intentions to use them.

\section{P4.083 PREVALENCE AND CORRELATES OF SEXUAL PARTNER CONCURRENCY AMONG PATIENTS ATTENDING A PUBLICLY-FUNDED STI CLINIC IN THE UNITED STATES}

doi:10.1136/sextrans-2013-051184.0981

'M P Carey, 'T E Senn, ${ }^{2} \mathrm{P}$ Coury-Doniger, ${ }^{2} \mathrm{M}$ A Urban, ${ }^{3} \mathrm{~K}$ B Carey. ${ }^{1} T$ The Miriam Hospital, Providence, RI, United States; ${ }^{2}$ University of Rochester, Rochester, NY, United States; ${ }^{3}$ Brown University, Providence, RI, United States

Background Partner concurrency, or overlapping sexual partnerships, is associated with the transmission of HIV and other STIs. In the United States, some urban populations report high rates of partner concurrency but relatively few U. S. studies have investigated correlates of partner concurrency empirically. The purpose of the present study was to investigate the prevalence and correlates of partner concurrency among urban patients attending a publiclyfunded STI clinic.

Methods Participants were 502 patients attending an STI clinic (41\% female; 69\% African American). They completed a computerised survey assessing demographic characteristics, partner concurrency, substance use, concurrency norms, environmental factors affecting concurrency (i.e., shortage of men), attitudes towards concurrency, and self-efficacy for having one partner.

Results Nearly one-half of the sample (46\%) reported concurrent sexual partnerships in the past 3 months. In univariate analyses, being male, drinking more, using marijuana more frequently, perceiving multiple partners as normative, perceiving that the shortage of men influenced sexual partnerships, more positive concurrency attitudes, and less self-efficacy for having one partner were associated with concurrency (all $p s<0.05$ ). Other demographic characteristics (race, income, education, age) were not associated with concurrency. In multivariate analyses, greater marijuana use $(\mathrm{OR}=1.17)$ and having more positive concurrency attitudes $(O R=1.95)$ were independently associated with partner concurrency ( $p s<0.05$ ).

Conclusion The prevalence of concurrency was high in this population of patients attending an STI clinic. Interventions to reduce concurrency should target marijuana use and attitudes towards concurrency.

\section{P4.084 SIGNIFICANT BEHAVIOUR CHANGE IN PEOPLE WHO INJECT DRUGS (PWID) AND FEMALE SEX WORKERS (FSWS) IN BANGLADESH}

doi:10.1136/sextrans-2013-051184.0982

${ }^{1} \mathbf{M}$ N Uddin, ${ }^{1} \mathrm{~F}$ Sultana, 'S Rasin, ${ }^{2} Y$ Siddiqua, ${ }^{1} \mathrm{~A}$ K Bosu, ${ }^{1} \mathrm{M}$ Amin, ${ }^{1} \mathrm{Z}$ Hossain. ${ }^{1}$ Save the Children, Dhaka, Bangladesh; ${ }^{2} T h e$ Nielsen Company (Bangladesh) Ltd., Dhaka, Bangladesh

Background The HIV prevalence in Bangladesh is still low with $<0.1 \%$ in general population and $<1 \%$ in most at risk populations (MARPs) though all the risk factors prevail like unsafe injecting practises and sex work, low condom use, taboos, social denial, illiteracy and a lack of awareness. With grants from the Global Fund, Save the Children has doubled the national coverage for people who inject drugs (PWID) and female sex workers (FSWs). Nearly 13,500 PWID and 29,000 FSWs are under the programme coverage.

Methods Save the Children provides essential services for PWID through 69 drop in centres (DICs) and for FSWs through 100 DICs and 10 outreach offices in 53 districts. The DICs provides them with sterile needle-syringe, access to detoxification, abscess management, condoms, STI services, general health services, referrals and information including bathing, resting and recreational facilities. Over 23 million needle/syringe and 3.5 million condoms for PWID and 55 million condoms for FSWs are distributed. The programme also provides information and services through a multi-level multi-channel approach to the general and vulnerable youth.

Results The percentage of PWID using sterile injecting equipment increased from 39\% (Baseline Survey, 2008) to 92\% in 2012 (Mid Term Survey, 2012). PWID using condom during the most recent sexual contact with a FSWs within last 12 months increased from $39 \%$ to $75.4 \%$. FSWs using condom during the most recent sexual contact increased from $63 \%$ to $95.5 \%$. Young people aged $15-24$ (potential clients of FSWs and vulnerable to drugs), who correctly identified at least two ways of preventing HIV, increased from 40.8\% (Baseline Survey, 2005) to $81.7 \%$.

Conclusion The programme contributed a lot to bring positive behaviour changes in PWID and FSWs. Further expansion and scale up will enable them for safer practises that will help the country retaining the low HIV prevalence. 